\title{
Hades, A Radiographic Simulation Code
}

\author{
M. B. Aufderheide, D. M. Slone, A. E. Schach von \\ Wittenau
}

This article was submitted to $27^{\text {th }}$ Annual Review of Progress in Quantitative Nondestructive Evaluation, Ames, lowa, July 16-21, 2000

\section{August 18, 2000}

U.S. Department of Energy

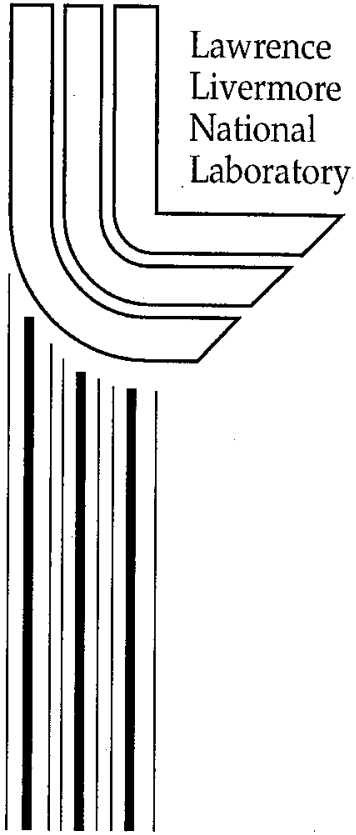




\section{DISCLAIMER}

This document was prepared as an account of work sponsored by an agency of the United States Government. Neither the United States Government nor the University of California nor any of their employees, makes any warranty, express or implied, or assumes any legal liability or responsibility for the accuracy, completeness, or usefulness of any information, apparatus, product, or process disclosed, or represents that its use would not infringe privately owned rights. Reference herein to any specific commercial product, process, or service by trade name, trademark, manufacturer, or otherwise, does not necessarily constitute or imply its endorsement, recommendation, or favoring by the United States Government or the University of California. The views and opinions of authors expressed herein do not necessarily state or reflect those of the United States Government or the University of California, and shall not be used for advertising or product endorsement purposes.

This is a preprint of a paper intended for publication in a journal or proceedings. Since changes may be made before publication, this preprint is made available with the understanding that it will not be cited or reproduced without the permission of the author.

This report has been reproduced

directly from the best available copy.

Available to DOE and DOE contractors from the

Office of Scientific and Technical Information

P.O. Box 62, Oak Ridge, TN 37831

Prices available from (423) 576-8401

http://apollo.osti.gov/bridge/

Available to the public from the

National Technical Information Service

U.S. Department of Commerce

5285 Port Royal Rd.,

Springfield, VA 22161

http://www.ntis.gov/

OR

Lawrence Livermore National Laboratory

Technical Information Department's Digital Library

http://www.llnl.gov/tid/Library.html 


\title{
HADES, A RADIOGRAPHIC SIMULATION CODE
}

\author{
Maurice B. Aufderheide, Dale M. Slone, Alexis E. Schach von Wittenau
}

Lawrence Livermore National Laboratory

\begin{abstract}
We describe features of the HADES radiographic simulation code. We begin with a discussion of why it is useful to simulate transmission radiography. The capabilities of HADES are described, followed by an application of HADES to a dynamic experiment recently performed at the Los Alamos Neutron Science Center. We describe quantitative comparisons between experimental data and HADES simulations using a copper step wedge. We conclude with a short discussion of future work planned for HADES.
\end{abstract}

\section{MOTIVATION}

Transmission radiography is used in many industrial settings for investigating parts or dynamic processes. It is useful to simulate the radiographic process for a number of reasons. First, an accurate simulation allows an honest comparison to be made between models and experimental results. Second, simulation can help the user understand features present in transmission radiographs, such as spectral hardening. Third, simulation can help the user to correct non-ideal effects in radiographs that might be input to tomography codes. Simulation is also a valuable aid for planning experiments so that the radiographic system is optimized. Another motivation for doing radiographic simulation is to couple simulation with tomography, in order to separate radiographic effects from the reconstruction of the object (see Martz et al. in this volume).

The user has two main options in performing radiographic simulation: Monte Carlo techniques, and ray-tracing techniques. Monte Carlo techniques allow the user to study the full physics of radiography using many simulation particles to interact with the object, including source generation and detector properties. In principle, this approach allows the user to include all the relevant processes for radiography: absorption, scattering, and secondary particle production. For X-Rays some Monte Carlo codes with such capability are MCNP[1], COG[2], TART[3], the integrated Tiger series[4], and EGS4[5]. Unfortunately, these codes are very slow and cumbersome in the simulation of radiographic images. For a 300 300 image with $1 \%$ Monte Carlo statistical fluctuations, at least $10^{9}$ particles must be transported through the system. While this is becoming feasible with modern massively parallel machines, such capability is not available for routine studies of radiography.

Ray-tracing techniques are very useful for studies requiring a fast turnaround time. In this approach, a bundle of rays connecting the radiographic source and detector is traced through the specified system. The total path length along each ray is computed and stored in each pixel of the simulated detector. Spectral effects, various instrumental blurs and dose conversion can be included in the calculation. Since the path length computation only occurs along each straight ray, scattering effects are not computed with such a code, although they can be included after the fact. Because of the greater simplicity of this technique, ray-tracing simulations are much faster than their Monte Carlo cousins and can be performed on workstations or fast PCs or Macs. XRSIM[6], SINDBAD[7] and HADES are examples of ray tracing radiographic simulation codes. 


\section{FEATURES OF HADES}

HADES is a ray-tracing code that we have been developing for the simulation of radiography used in industrial NDE settings. The name HADES was derived from Greek mythology. For the ancient Greeks, Ai $\delta\rceil \zeta$ was the underworld, the abode of the dead. In old-fashioned English translations of the Greek classics, the dead in At $\delta \eta$ swere referred to as shades, or shadows. Since radiography is the science of making and interpreting shadows, HADES was considered an appropriate name for a radiographic simulation code.

For HADES, the fundamental object description that is radiographed is a meshed model of an object. By mesh, we do not mean the surface meshes often discussed in computer graphics[8,9], but rather a finite-element or finite-difference mesh, which describes the volume of the object. HADES can radiograph $2 \mathrm{D} \mathrm{r}-\mathrm{z}$ meshes of varying types, as well as 3D meshes of complexity ranging from Cartesian meshes to unstructured generalized hexahedral meshes.

HADES also has a library of solid-body objects such as plates, spheres, cones, cylinders and other complex shapes that can be included in a simulation. Recently we have added constructive solid geometry operations such as intersections, unions and differences to allow more complex objects to be built and radiographed. HADES is often run without any mesh, only using combinations of solid body objects.

Because we use a wide variety of radiographic probes at Livermore, we have given HADES the capability to support these probes. HADES can simulate X-Ray radiography for photon energies ranging from roughly $\sim 1 \mathrm{keV}$ to $100 \mathrm{MeV}$. Spectral and monochromatic sources can be simulated by the code. HADES uses the Livermore Evaluated Photon Data Library[10] for X-Ray absorption cross-sections. HADES can also simulate neutron radiography for neutron energies ranging from roughly thermal energies up to $30 \mathrm{MeV}$. HADES uses the Livermore Evaluated Neutron Data Library[11] for these simulations. Again, the user can specify spectral or monochromatic neutron sources. HADES can also simulate high-energy proton radiography for proton energies ranging from $\sim 800 \mathrm{MeV}$ up to $\sim 100 \mathrm{GeV}$, taking into account Gaussian multiple Coulomb scattering. For this work, HADES uses the Letaw nuclear attenuation cross-sections [12] and Dahl s expression for radiation length[13].

One of our goals in the development of HADES has been to accurately simulate the radiographic process, from source to image formation and detection. Thus the code simulates source blur either approximately by projecting this blur onto the detector plane, or by mapping out the finite spot by brute force. Detector blur and spectral response can be included in the simulation several different ways. The user can include a scattering profile in the simulation, but HADES does not independently compute scattering profiles. This computation must be either estimated analytically or computed with a Monte-Carlo code.

We currently use POVRay[14] to render our radiographic systems, in order to ensure that the system is what the user desired. We are now moving to $v t k[15]$ because of its greater functionality and will soon be rendering with it.

Some parts of the code are parallelized, in order to simulate finite spot size by brute force and to speed up computationally intense simulations, such as ray tracing $3 \mathrm{D}$ meshes. This work has been reported elsewhere[16]. HADES currently runs on SGI and Compaq workstations, as well as Cray supercomputers and the ASCI[17] Blue Pacific machine.

In Figure 1 on the next page, we display a set of pencils, marbles, a key, and a ring embedded in some concrete. We show the rendering of the system, and simulated 
radiographs using $4 \mathrm{MeV}$ X-Rays, $10 \mathrm{MeV}$ neutrons, and $800 \mathrm{MeV}$ protons with a 10 milliradian angular cut applied. With X-Rays the higher density objects (key, ring, and marbles) are much more opaque than the lower density objects (wood, graphite, and rubber). With neutrons, the situation is different, because they preferentially scatter off hydrogen and low $\mathrm{Z}$ materials. In this case, the dynamic range of the pencils is more similar to the dynamic range for the metal pieces. We see a mixture of these effects with protons. Being hadrons, protons attenuate in a manner similar to neutrons. But protons Coulomb scattering results in an angular attenuation that is similar to what is seen with X-Rays. In this image, the angular attenuation has a larger effect than the nuclear attenuation. Switching from one radiographic probe to another only required changing a few lines of the input instructions to HADES.

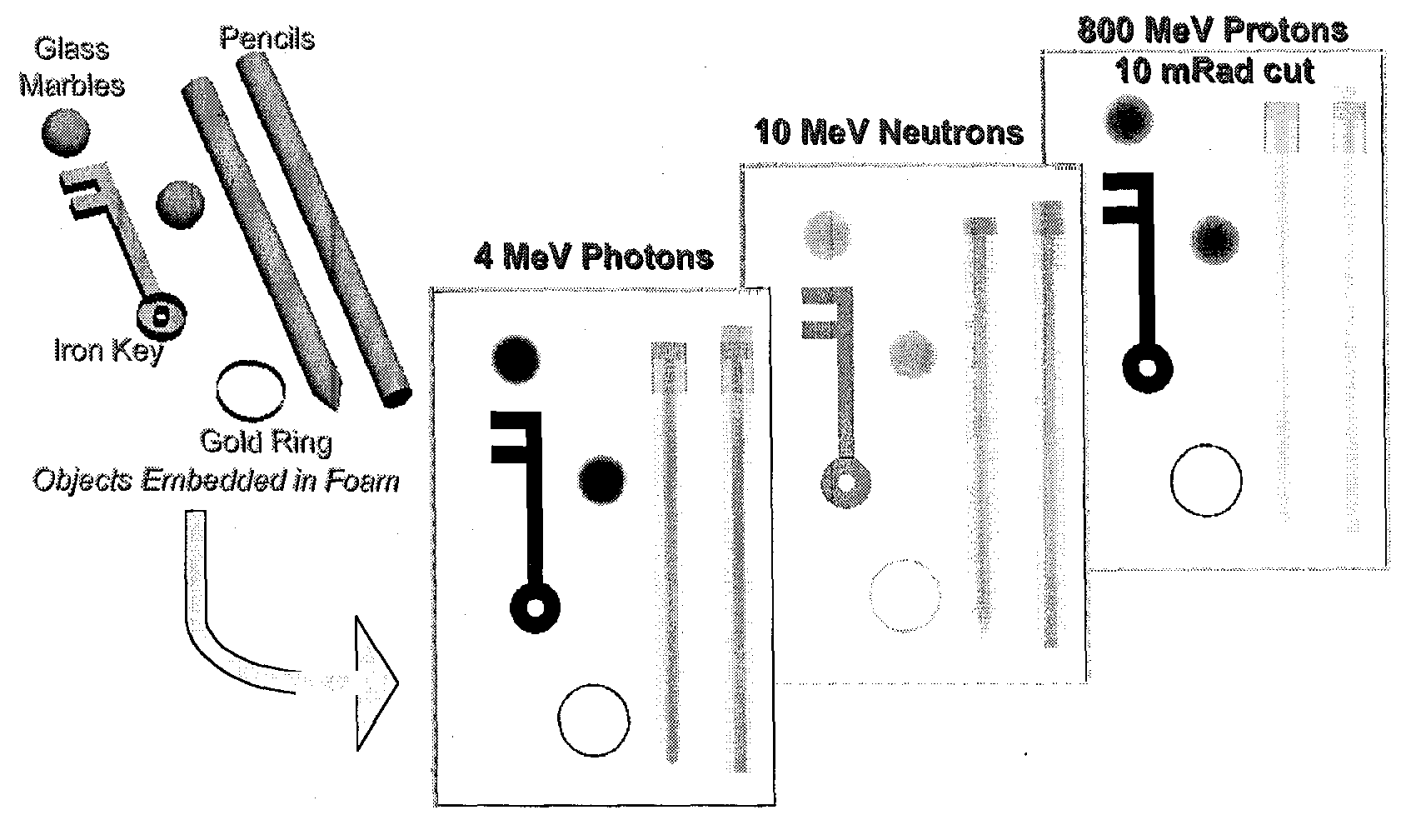

Figure 1: HADES simulated radiographs through a set of solid body objects. The radiographic probes shown are $4 \mathrm{MeV}$ photons, $10 \mathrm{MeV}$ neutrons, and $800 \mathrm{MeV}$ protons with a 10 mRadian angular cut. POV-Ray was used to render the system as shown at the upper left of the figure.

\section{MESH RAY TRACING EXAMPLE: PROTON RADIOGRAPHY}

As an example of ray tracing through meshes, we will discuss the Hopyard radiographic experiment done recently at the Los Alamos Neutron Science Center (LANSCE). In this experiment, we wanted to study the response of tin to a violent event such as shock wave. A $4 \mathrm{~cm}$ diameter tin disk was mounted on a sandwich of $8 \mathrm{~cm}$ diameter aluminum plates, which had a high explosive between them.

The system before detonation of the high explosive and $12.26 \mu$ s later are displayed in Figure 2 on the next page. The detonation is initiated at the bottom explosive/aluminum interface. At the later time, the detonation front has finished burning through the explosive and the front has hit the upper aluminum plate very hard, generating a shock in the metal. This shock breaks up the tin into spall layers.

The upper row of Figure 2 shows cross sections of the density within the object as calculated by CALE, a 2D hydrodynamics code developed at LLNL. In the middle row. of Figure 2 are the actual radiographs taken during the experiment. 
On the bottom row are HADES simulations of the experimental data. HADES produced these images by tracing through the 200x500 $r-z$ mesh that the hydro code had produced. This requires that, for each ray to a detector pixel, all intersections of the ray with zone boundaries be computed and total path lengths be added. Since the density can be different in each zone, the path length must be computed zone by zone along the track. This kind of mesh is challenging because the vertices (the intersections of the $z$ and $r$ lines) do not necessarily form right angles between the $z$ and $r$ lines. Such generality has made ray tracing through this mesh fairly difficult to do reliably.
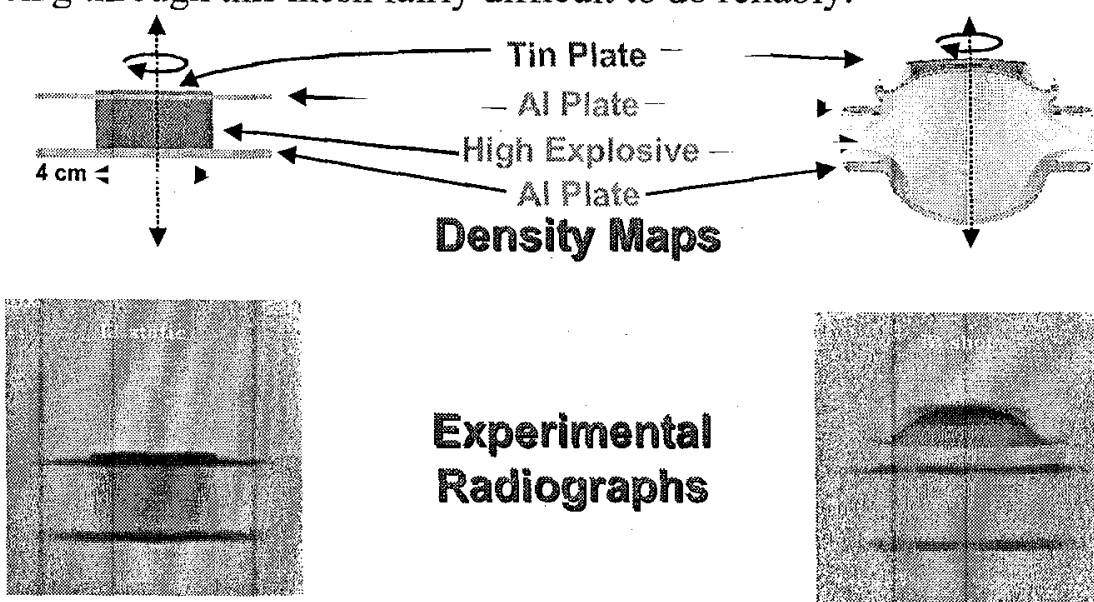

\section{Experimental} Radiographs
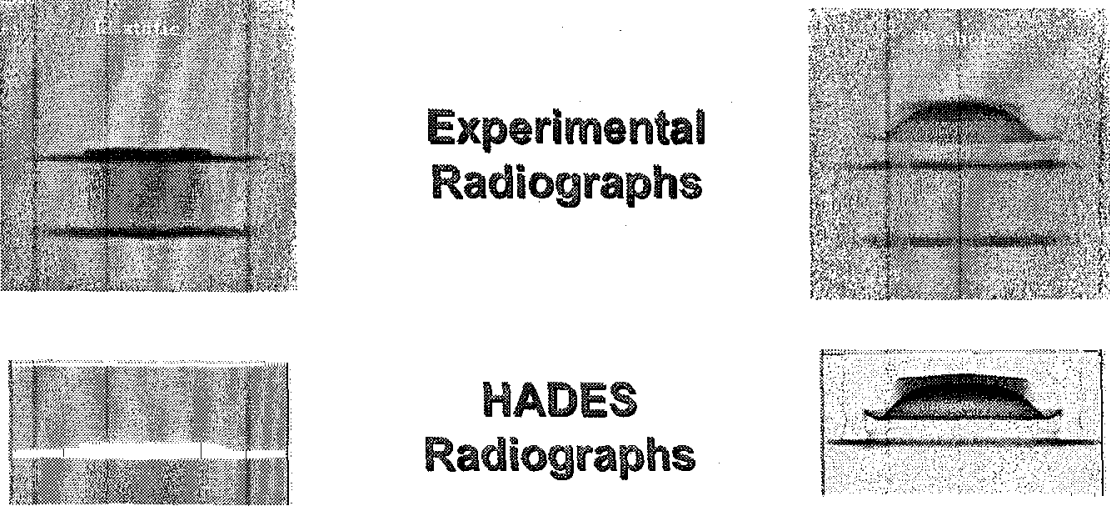

\section{Initial Configuration}

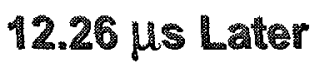

Figure 2: Comparison between actual proton radiographs and simulations produced by HADES, ray tracing through meshes produced by the CALE hydrodynamics code.

It can be seen that there is not perfect agreement between the simulations and the data, but the agreement is strikingly good. We are presently investigating the sources of these differences. One difference may be in the timing. The simulation appears to be somewhat late, compared with the data. Another source of differences may be the cross sections used. The Letaw cross sections were derived as fits to a limited data set and thus may be inaccurate. Step wedge images were also imaged in this experiment and will help to determine the correct cross sections for these objects.

As mentioned above, HADES supports a variety of meshes, so that a variety of hydrodynamic calculations can be radiographed. The largest meshes that we have radiographed contained on the order of 100 million zones. The size of these problems will increase in the future. For such large calculations, HADES must be run in parallel to provide a simulation in a reasonable time. When run in parallel on such large jobs, HADES takes on the order of an hour of wall clock time to complete a simulation.

\section{QUANTITATION: SIMULATION OF A COPPER STEP WEDGE}

Another aspect of our work on HADES has been efforts to provide simulations that are quantitatively correct, not just similar to experimental data. We need quantitative accuracy because of our goals to use HADES for experiment planning and optimization, 
and also for coupling with optimization codes for tomography (see the talk by Martz et al. in this volume).

We have chosen LLNL s $9 \mathrm{MeV}$ Linac as our X-Ray source for these studies and for detectors have been using MinR screens plus dpi $\chi$ large format amorphous silicon arrays. We have been using a 14 step copper step wedge to test our ability to simulate the correct amount of attenuation in the system. The step thicknesses in the system vary from roughly $0.34 \mathrm{~cm}$ to $10.2 \mathrm{~cm}$. Because of the large size of the object relative to the imaging array, relatively low magnifications were used. Magnifications ranged from nearly 1 to 1.2 . The source to object distance was $5 \mathrm{~m}$. In Figure 3, we display the step wedge and its experimental radiograph, when magnification was 1.2.

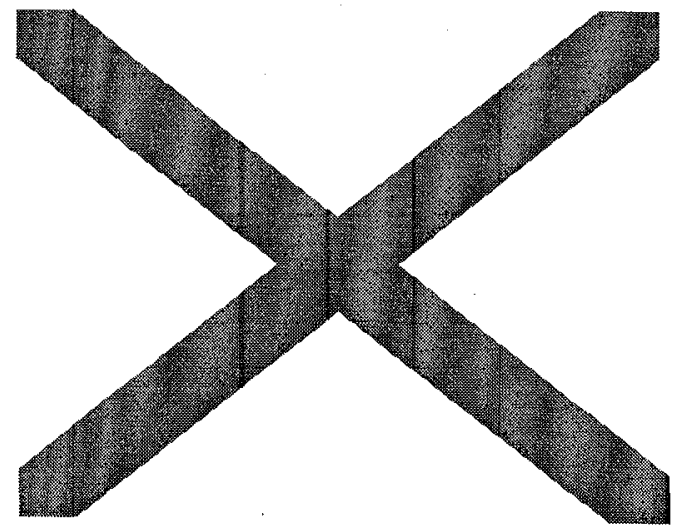

Figure 3: Using a copper step wedge to quantify HADES simulation capabilities. The upper row shows the step wedge and its radiograph, while the bottom row shows lineouts across the center of the step wedge radiograph and the HADES simulation. The plot at the bottom left illustrates that a poor fit is obtained if detector response is not included, while the plot at the right shows how detector response improves the simulation s fit to the data.

In the figure, we also show a lineout across the step wedge and compare it with two HADES simulations. In the first comparison, HADES included the $9 \mathrm{MeV}$ bremsstrahlung spectrum in its simulation, but the detector response was not modeled. In the second comparison, the energy dependent blur of the dpi $\chi$ detector were included in the simulation. The second simulation shows excellent agreement to the blurring seen in the radiograph. Agreement between simulation and experiment are currently at the 3\% level. We anticipate that even better agreement will be seen when the finite spot size is included in the simulation. In these simulations, the scattered background was treated as a uniform value that was determined by optimizing the fit to the data. We are trying to quantify this part of the problem better. 